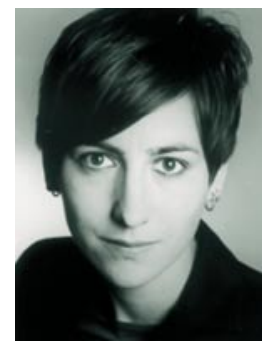

Barbara Hoefgen

\title{
Genetische Grundlagen der Schizophrenie
}

\author{
Barbara Hoefgen ${ }^{1}$, Marcella Rietschel', Manfred Ackenheil'2, Wolfgang Maier ${ }^{1}$ \\ ${ }^{1}$ Klinik und Poliklinik für Psychiatrie und Psychotherapie der Universität Bonn \\ 2 Klinik für Psychiatrie und Psychotherapie der Ludwig-Maximilians-Universität München
}

psychoneuro 2004; 30 (11): 603-605

$\mathrm{E}$ rste Erkenntnisse zur Vererbbarkeit schizophrener Erkrankungen wurden bereits um die Jahrhundertwende von Emil Kraepelin aufgezeigt und konnten in der Folgezeit durch systematische Familienuntersuchungen bestätigt werden. Das Erkrankungsrisiko korreliert dabei stark mit dem Verwandtschaftsgrad, aber auch mit dem Schweregrad der Erkrankung des betroffenen Familienmitglieds. Mittlerweile wird davon ausgegangen, dass Umwelteinflüsse und genetische Faktoren gemeinsam zur Entstehung schizophrener Erkrankungen beitragen. Wie hoch der jeweilige Beitrag ist oder auf welche Weise beide Bereiche zusammenwirken (additiv und/oder interagierend), ist bisher noch unklar. Hinweise auf die Beteiligung von genetischen Faktoren liefern Familien-, Zwillingsund Adoptionsstudien. Eine Zusammenfassung der bisherigen Zwillingsuntersuchungen durch Gottesman (3) ergab eine Konkordanzrate von etwa $50 \%$ bei eineiigen Zwillingen. Da für schizophrene Erkrankungen weder ein Erbgang mit monogener, dominanter (Beispiel: Chorea Huntington) oder rezessiver Vererbung (Beispiel: zystische Fibrose) nachgewiesen werden konnte, werden schizophrene Erkrankungen den komplexen multifaktoriellen genetischen Erkrankungen mit oligo- bzw. polygener Vererbung zugeordnet. In Abbildung 1 ist die hypothetische Beziehung zwischen Dispositionsgenen

Schizophrene Psychosen sind zu ungefähr 50\% genetisch bedingt. Die Beteiligung genetischer Faktoren konnte durch Familien-, Zwillings- und Adoptionsstudien nachgewiesen werden. Da sich diese genetische Komponente nicht durch den Beitrag eines einzelnen, kausalen Gens erklären lässt, werden schizophrene Psychosen den komplexen genetischen Erkrankungen mit oligo- oder polygener Vererbung und einer gleichzeitigen Beteiligung von Umweltfaktoren zugeordnet. Fortschritte der molekulargenetischen Forschung konnten erheblich zur Entschlüsselung der Ursachen schizophrener Psychosen beitragen. Mehrere Regionen auf dem menschlichen Genom sind mittlerweile identifiziert, in denen Dispositionsgene für die Erkrankung liegen. Dieser Beitrag soll eine Einführung in die theoretischen Grundlagen und einen Überblick über die wichtigsten bisherigen Ergebnissen geben sowie Ziele und Ergebnisse des speziellen Projektverbunds „Molekular- und Pharmakogenetik“ des Kompetenznetzes Schizophrenie vorstellen.

und dem Phänotyp der Erkrankung dargestellt.

$\mathrm{Zu}$ den wesentlichen Voraussetzungen für die Aufklärung der genetischen Ursachen der Schizophrenie zählen die Verfügbarkeit der DNA einer möglichst großen Anzahl von an schizophrenen Störungen betroffenen, nicht miteinander verwandten Personen (Assoziationsstichprobe) bzw. von Familien, in denen die Erkrankung vererbt wird (Kopplungsstichprobe), sowie die jeweils gesicherte psychiatrische Diagnose der beteiligten Personen. Bei beiden Stichproben wird untersucht, ob genetische Varianten häufiger bei den Erkrankten nachgewiesen werden können als durch Zufall zu erwarten ist.

Bei der Kopplungsanalyse wird untersucht, ob die gemeinsame Vererbung einer DNA-Variante häufiger an erkrankte Familienmitglieder erfolgt. Die Variante, deren Lokalisierung bekannt sein muss, kann in die- sem Fall Aufschluss über die bisher unbekannte Lokalisation des Krankheitsgenorts geben. In Assoziationsstudien wird überprüft, ob ein statistisch signifikanter Unterschied in der Verteilung der Allele eines Polymorphismus in Stichproben aus nicht verwandten Erkrankten im Vergleich mit Kontrollpersonen besteht.

In den letzten 20 Jahren wurde weltweit eine Vielzahl von Kopplungs- und Assoziationsstudien durchgeführt. Zurzeit werden Genregionen für schizophrene Erkrankungen auf folgenden Chromosomen diskutiert (7): 1q, 5q, 6p, 6q, 8p, 10p, 13q und 22q.

Ausgehend von der systematischen Genortsuche wurden in letzter Zeit mit Hilfe von Assoziationsuntersuchungen Kandidatengene nachgewiesen, die als Dispositionsgene in Frage kommen (6). Zu diesen Kandidatengenen gehören:

- Dysbindin auf Chromosom 6: Die von der Arbeitsgruppe 
Abb. 1 Hypothetische Beziehung zwischen Dispositionsgenen und dem Phänotyp der Erkrankung

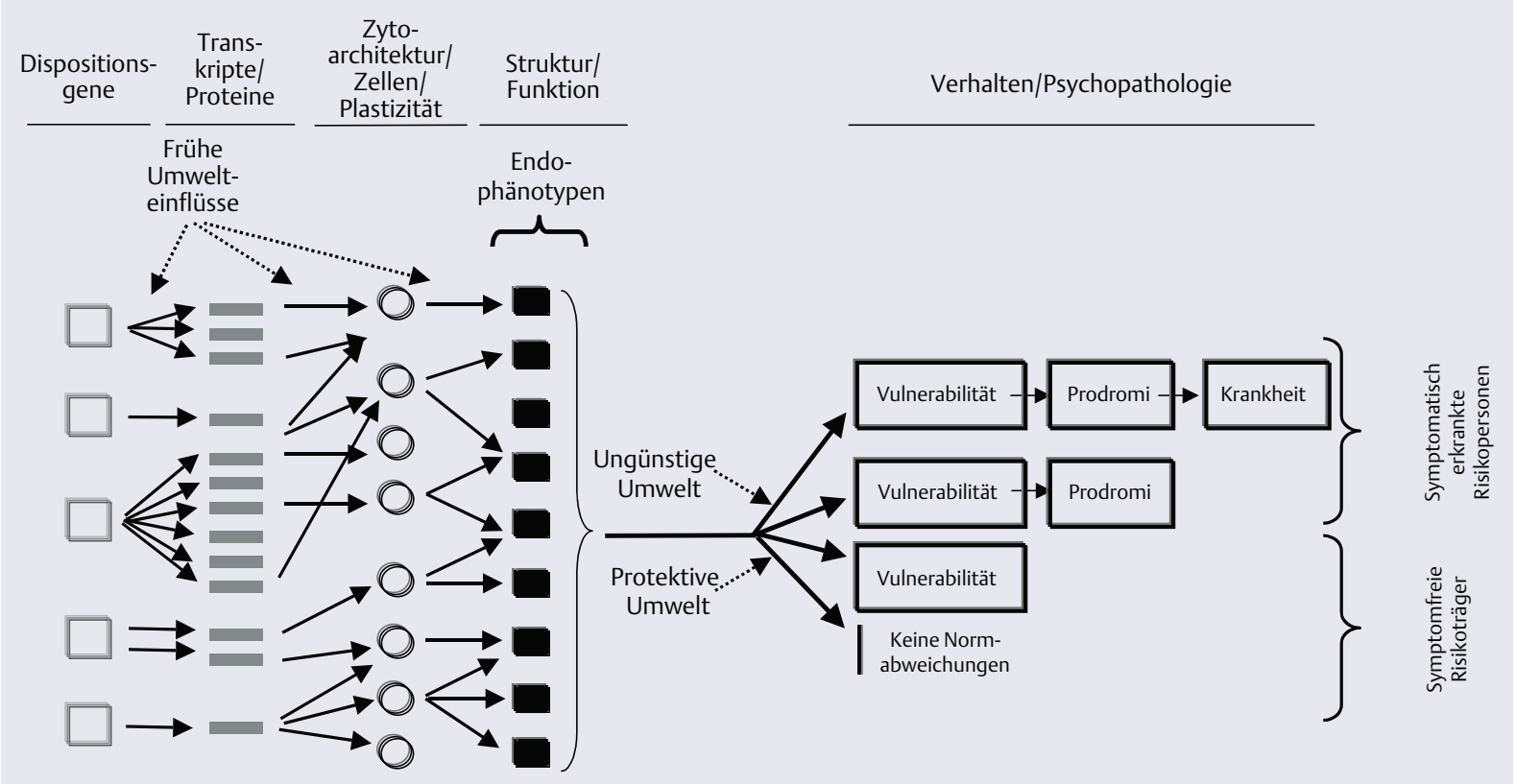

Genvarianten in unterschiedlicher Zusammensetzung und Menge prägen zusammen mit frühen Umweltfaktoren den Endophänotyp. Dieser ist wiederum für die Ausbildung des psychopathologischen Phänotyps mitverantwortlich, wobei Umwelteinflüsse sowohl verstärkend als auch abschwächend beitragen können.

Straub et al. gefundene Assoziation (13) konnte auch von unserer eigenen Arbeitsgruppe in zwei unabhängigen Familienstichproben bestätigt werden (10). Dysbindin spielt möglicherweise eine strukturelle Rolle bei der Bildung und dem Erhalt von neuromuskulären Synapsen

- Neuregulin-1 auf Chromosom 8: Etwas oberhalb der Region, in der positive Kopplungsbefunde von mehreren Arbeitsgruppen erhalten wurden $(4,8)$, konnte nun das Neuregulin-1-Gen nachgewiesen werden, welches in unabhängigen Stichproben Assoziation mit Schizophrenie zeigt (11, 12). Neuregulin spielt eine Rolle in Expression und Aktivierung von Neurotransmitterrezeptoren (u.a. Glutamatrezeptoren)

- G72 auf Chromosom 13: Angrenzend an die Region, in der Hinweise auf ein Suszeptibilitätsgen erhalten wurden, konnte ein Gen (G72) mit Assoziation zur Schizophrenie nachgewiesen werden (1). Der Bonner Arbeitsgruppe gelang es, diesen Assoziationsbefund zu replizieren (9)

- Weitere Kandidatengene aus den diskutierten Genregionen sind
COMT (Catechol-O-MethylTransferase), DISC1 (DisruptedIn-Schizophrenia 1) und PRODH (Prolin-Dehydrogenase).

Alle diese Gene sind in biologische Mechanismen involviert, von denen angenommen wird, dass sie bei der Schizophrenie gestört sind (z.B. synaptische Transmission, Signaltransduktion, Neuroentwicklung, Neurotransmitterregulation). Die pathogenen Mutanten der drei identifizierten möglichen Dispositionsgene und deren funktionelle Konsequenzen in Bezug auf die Krankheitsentstehung sind momentan noch nicht bekannt. Soweit derzeit absehbar, haben diese Gene aber recht wenig mit jenen Wirkmechanismen zu tun, die der Entwicklung der für die Behandlung der Schizophrenie eingesetzten Pharmaka, den Neuroleptika, zu Grunde liegen. Obwohl die antipsychotischen Effekte dieser Medikamente gut belegt sind, ist ihre Wirkung doch unzureichend, sodass neue psychopharmakologische Behandlungsverfahren mit neuen Wirkmechanismen für eine bessere Behandlung der betroffenen Patienten dringend erforderlich wären. Die nun neu identifizierten
Dispositionsgene für die Schizophrenie stellen hoffnungsvolle Kandidaten für die Entwicklung neuer Wirkprinzipien bei der Behandlung der Schizophrenie dar.

\section{Spezieller Projektverbund „Molekular- und Pharmako- genetik“"}

Ziel dieses Projektes des Kompetenznetzes Schizophrenie ist die Erforschung einer Genotyp-PhänotypBeziehung mit der Fragestellung, wie klinische oder neurobiologische Krankheitskorrelate durch spezifische Dispositionsgene beeinflusst werden.

Des Weiteren sollen genetische Determinanten identifiziert werden, die einen Beitrag leisten zu den individuellen Unterschieden von Krankheitsentstehung und -verlauf, Ansprechen auf Medikamente und Nebenwirkungen, Plasmaspiegel (Pharmakokinetik), Wirkweise an den Rezeptoren (Pharmakodynamik) und Modulation der Genexpressionsstärke.

Für diesen Zweck ist in Bonn und München eine langfristig angelegte Zell-/DNA-Bank für Schizophrenie mit klinisch, neurobiologisch und pharmakologisch charakterisiertem Phänotyp angelegt worden. Dazu 
sind Blutproben von Patienten, die an den unterschiedlichen Projekten in 14 deutschen psychiatrischen Kliniken teilgenommen haben, innerhalb des Netzwerks gesammelt worden. Bislang konnten 390 Patienten in das Projekt eingeschlossen werden. Durch das Netzwerk ist somit die Möglichkeit geschaffen worden, genetische Faktoren an einem einzigartigen Patientenkollektiv.

Zunächst wurde eine Vielzahl von genetischen und pharmakogenetischen Untersuchungen in Kollektiven durchgeführt, die in den Zentren Bonn und München rekrutiert worden sind. Dabei wurden Polymorphismen untersucht in den Genen für den Serotonintransporter (5HTT), für die Serotoninrezeptoren 5HTR2a und 5HTR2c, für die Dopaminrezeptoren DRD2 und DRD3, für die G-Protein-Untereinheiten Gß3 und GOLF, für die Monoaminooxidase A (MAOA) sowie für COMT, ACE (Angiotensin Converting Enzyme), BDNF (Brain-Derived Neutrophic Factor), Dysbindin, NOTCH4 (Homolog des NOTCH-Gens), G72, DAAO, MDR1 (Multidrug Resistance 1) und das Cytochrom P450-System.

In der Bonner Arbeitsgruppe konnten durch Schwab et al. (10) in zwei unabhängigen Familienkollektiven die von Straub et al. (13) beschriebene Beteiligung des Dysbindin-Gens an der Ätiologie schizophrener Psychosen bestätigt werden. Weiterhin gelang es Schumacher et al. (9), die von Chumakov et al. (1) berichtete Assoziation zwischen genetischen Varianten im G72-Gen sowie im D-Amino-AcidOxidase-Gen (DAAO-Gen) mit Schizophrenie zu replizieren.

Darüber hinaus wurden pharmakogenetische Untersuchungen zur Clozapin-Response und zum Auftreten tardiver Dyskinesien durchgeführt. Im Hinblick auf das Auftreten tardiver Dyskinesien ist in der Einzelanalyse die Anzahl der Patienten nicht ausreichend, um eine Assoziation des Dopamin-D3-RezeptorSer9Gly-Polymorphismus mit dem Auftreten tardiver Dyskinesien zu zeigen. In einer Metaanalyse werden die Befunde jedoch signifikant (5).

Replikationsstudien im Gesamtsample dieses Projektes werden nun insbesondere an diesen Genen sowie am Neuregulin-1-Gen und weiteren, möglicherweise neu beschriebenen Kandidatengenen durchgeführt. Zusätzlich zu den Genotypen sollen auch Risikohaplotypen in Dispositionsgenen, welche mit der $\mathrm{Er}$ krankung assoziert sind, mit klinischen, neuropsychologischen und -morphologischen Merkmalen (Endophänotypen) korreliert werden. Erweiterte Stichproben mit neurobiologischen und Therapie-bezogenen Phänotypen ersterkrankter schizophrener Patienten eröffnen die einzigartige Möglichkeit, einen sehr viel genaueren Zusammenhang zwischen Genotyp und Phänotyp aufzustellen. Ein möglicher Ansatzpunkt wäre z.B. das Arbeitsgedächtnis, dessen Leistungsfähigkeit in einer Studie als assoziiert mit einem Met/Val-Polymorphismus im COMTGen (2) gefunden wurde. Sobald die klinisch erhobenen Daten in einer netzwerkinternen zentralen Datenbank zusammengeführt sind, sind auch die ersten Ergebnisse zu Genotyp-Phänotyp-Beziehungen zu erwarten.

\section{Genetic basis of schizophrenia}

Genetic determination and heritability of schizophrenic disorders is evidenced by family, twin and adoption studies. It is clear from studies on different classes of relatives that the recurrence risk decreases too rapidly with increasing genetic distance from the proband for schizophrenia to be a single-gene disorder. The mode of transmission is rather like that of a complex and non-Mendelian common disorder. Progress in development of methods in molecular genetics contribute to the search for causes of the disease. Recently, several disposition genes for schizophrenia were identified. This review gives a short introduction into the theoretical background and presents the results of genetic causes of schizophrenia and the aims and results of subproject "molecular-/ pharmacogenetics" of the German Research Network on Schizophrenia.

\section{Key Words}

schizophrenia genetics - linkage association studies - disposition genes

\section{Literatur}

1. Chumakov I, Blumenfeld M et al. Genetic and physiological data implicating the new human gene $G 72$ and the gene for $D$ amino acid oxidase in schizophrenia. Proc Natl Acad Sci U S A 2002, 99:13675-13680

2. Egan MF, Goldberg TE et al. Effect of COMT Val108/158Met genotype on frontal lobe function and risk for schizophrenia. Proc Natl Acad Sci U S A. 2001; 98(12): 6917-6922

3. Gottesman I, Schizophrenia Genesis. New York: WH Freeman 1991

4. Kendler KS, MacLean CJ et al. Evidence for a schizophrenia vulnerability locus on chromosome 8p in the Irish Study of HighDensity Schizophrenia Families. Am J Psychiatry 1996; 153: 1534-1540

5. Lerer B, Segman RH et al. Pharmacogenetics of tardive dyskinesia: combined analysis of 780 patients supports association with dopamine D3 receptor gene Ser9Gly polymorphism. Neuropsychopharmacology 2002; 27: 105-119

6. Maier W, Zobel A et al. Genetics of schizophrenia and affective disorders. Pharmacopsychiatry 2003; 36 (Suppl 3): 195-202

7. Owen MJ, Williams NM et al. The molecular genetics of schizophrenia: new findings promise new insights. Mol Psychiatry 2004; 9: 14-27

8. Pulver AE, Lasseter VK et al. Schizophrenia: a genome scan targets chromosomes $3 p$ and $8 p$ as potential sites of susceptibility genes. Am J Med Genet 1995, 60: 252-260

9. Schumacher J, Jamra RA et al. Examination of G72 and D-amino-acid oxidase as genetic risk factors for schizophrenia and bipolar affective disorder. Mol Psychiatry 2004, 9: 203-207

10. Schwab SG, Knapp M et al. Support for association of schizophrenia with genetic variation in the 6 p22.3 gene, dysbindin, in sib-pair families with linkage and in an additional sample of triad families. Am J Hum Genet 2003; 72: 185-190

11. Stefansson H, Sarginson J et al. Association of neuregulin 1 with schizophrenia confirmed in a Scottish population. Am J Hum Genet 2003, 72: 83-87

12. Stefansson H, Sigurdsson E et al. Neuregulin 1 and susceptibility to schizophrenia. Am J Hum Genet 2002; 71: 877-892

13. Straub RE, jiang $Y$ et al. Genetic variation in the 6p22.3 gene DTNBP1, the human ortholog of the mouse dysbindin gene, is associated with schizophrenia. Am J Hum Genet 2002; 71: 337-348

\section{Korrespondenzadresse:}

Dr. rer. nat. Barbara Hoefgen

Klinik und Poliklinik für Psychiatrie

und Psychotherapie

Universität Bonn

Sigmund-Freud-Str. 25

53105 Bonn

Barbara.Hoefgen@ukb.uni-bonn.de 